\title{
Intramural duodenal hematoma post EUS-guided placement of fiducial radiopaque markers
}

Ihab I. El Hajj, MD, MPH, ${ }^{1}$ Jeffrey J. Easler, MD,${ }^{1}$ Stuart Sherman, MD, ${ }^{1}$ Mohammad Al-Haddad, MD, ${ }^{3}$

${ }^{1}$ Department of Internal Medicine, Division of Gastroenterology and Hepatology, Section of Interventional Endoscopy, Indiana University School of Medicine, Indianapolis, IN

\author{
Corresponding author: \\ Ihab I. El Hajj, MD, MPH \\ Assistant Professor of Medicine \\ Indiana University School of Medicine \\ 550 N University Blvd, Suite 1634E \\ Indianapolis, IN 46204 \\ Phone: 317-274-3255 \\ Fax: 317-948-0164 \\ Email: ielhajj@iu.edu
}

\section{Case}

A 61-year-old man presented with painless jaundice. Abdominal CT showed head of the pancreas mass. EUS-FNA confirmed adenocarcinoma. ERCP was performed with sphincterotomy/stent placement. Case was reviewed at our pancreas tumor board: borderline resectable tumor, recommended upfront neoadjuvant chemoradiation. Patient was referred for EUS-guided fiducial placement before stereotactic body radiation therapy (SBRT). With the linear echoendoscope positioned in the duodenal lumen, a preloaded fiducial in a $22 \mathrm{G}$ needle EchoTip Ultra Fiducial Needle (Cook Medical; Bloomington, IN) was deployed in the tumor. Maneuver was repeated for a total of 3 fiducials (Fig. 1, black arrows). The patient presented 2 days later with abdominal pain/bilious vomiting. Abdominal CT: no pneumoperitoneum/pancreatitis, markedly distended stomach with fluid retention suggestive of gastric outlet obstruction, and circumferential/diffuse wall thickening/intramural hyperdensity/luminal narrowing of the duodenal bulb/proximal D2 suggestive of intramural duodenal hematoma (IDH) (Fig. 2A\&B). The patient was managed conservatively. An 18Fr nasogastric tube was placed and connected to low intermittent suction. His symptoms gradually improved over 4-5 days. Upper GI series at Day-6 confirmed the absence of residual mechanical obstruction (Fig. 2C).

This is the author's manuscript of the article published in final edited form as:

El Hajj, I. I., Easler, J. J., Sherman, S., \& Al-Haddad, M. (2017). Intramural duodenal hematoma post EUS-guided placement of fiducial radiopaque markers. Digestive and Liver Disease. https://doi.org/10.1016/j.dld.2017.08.028 
Fiducials are radiopaque markers implanted into a cancer lesion for localization and accurate treatment delivery during SBRT.

EUS-guided fiducial placement +/- fluoroscopy guidance, permits placement of fiducials into lesions in close proximity to the GI tract and often in locations that are difficult to access with alternative methods. Several series reported safe and successful EUS-guided implantation of fiducials into primary or metastatic pancreatic cancer, among many other cancers. This is the first report of IDH post EUS-guided fiducial placement. A combination of echoendoscope torquing, and repeated punctures of a hypervascular pancreatic tumor through the duodenal wall may have contributed to this complication. IDH generally resolves with conservative management, occasionally requiring percutaneous, endoscopic or surgical drainage [1].

\section{Disclosure:}

Financial disclosure: None to report.

Informed consent was obtained for this case report.

Financial disclosure: None to report.

Informed consent was obtained for this case report.

Conflict of interest: None to report. 


\section{References}

[1] Pan YM, Wang TT, Wu J, et al. Endoscopic drainage for duodenal hematoma following endoscopic retrograde cholangiopancreatography: a case report. World J Gastroenterol 2013;19:2118-21. 
Fig 1

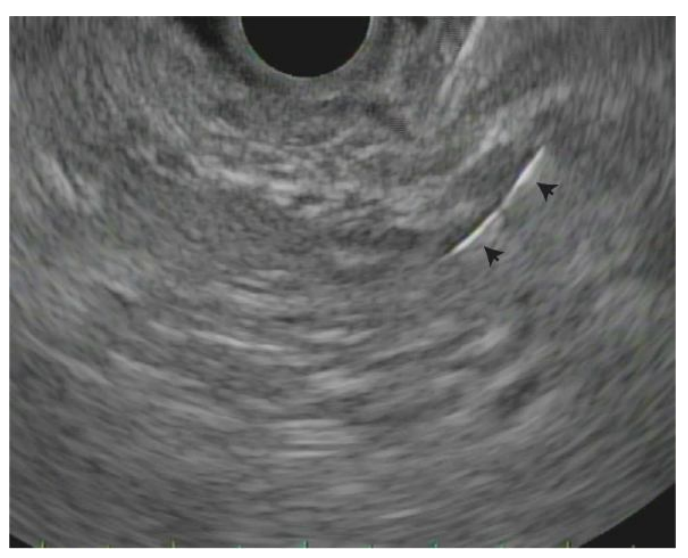

Fig $2 a$

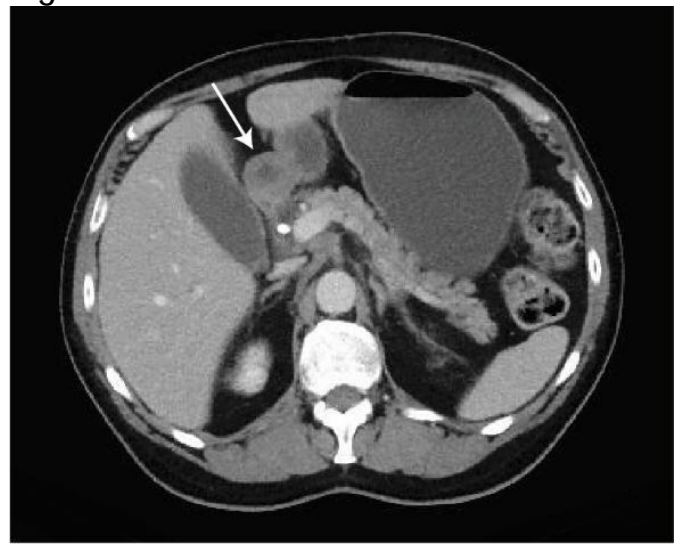

Fig 2 b

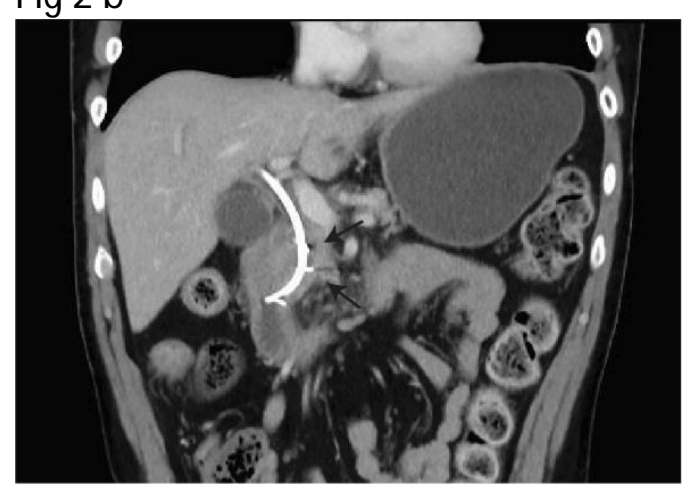

Fig $2 C$ 


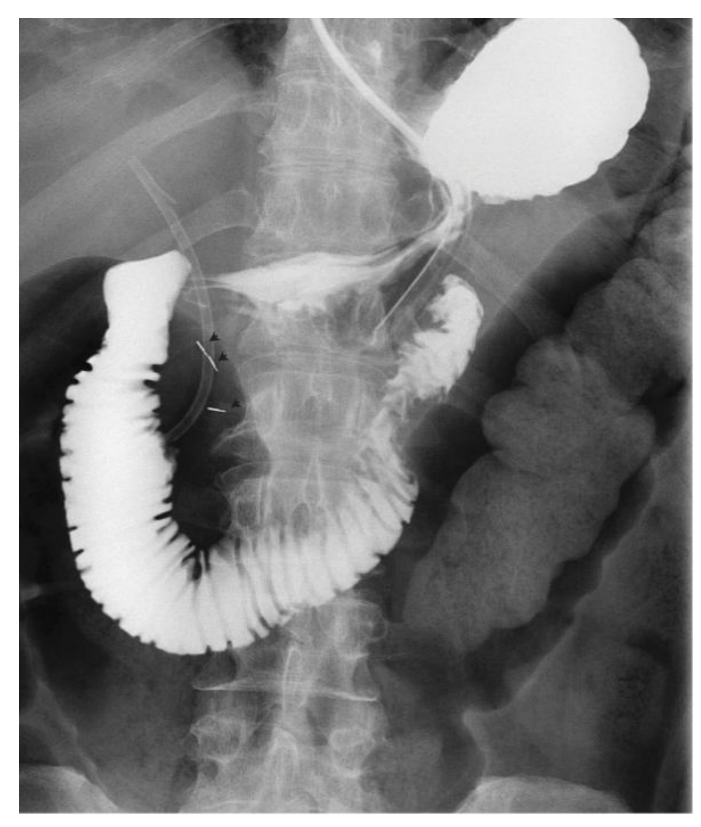

